\title{
Epidemiology and the Italian national health service
}

\author{
Paolo Vineis, Eugenio Paci
}

\begin{abstract}
The Italian national health service is under criticism. Although recent legislative changes are intended to introduce managed competition, there is no clear epidemiological view of the health care needs and priorities. Several inconsistencies and inequalities (particularly between the northern and the southern regions) are obvious, both in the health status of the population and in the provision of health care. Problems that should be addressed by future governments are: the uneven distribution of medical technology; the persistence of large inequalities, documented by outcomes such as rheumatic fever, between northern and southern regions; the large variability in clinical practices; the lack of an explicit policy as far as the diffusion of advanced technology is concerned; the ensuing "hidden" rationing; and the peculiar relationship between public and private health structures. Both the current ideological shift towards a market economy for health care and the traditional lack of epidemiological input in health care planning could threaten a rational approach to these problems.
\end{abstract}

( 7 Epidemiol Community Health 1995;49:559-562)

The 1978 law which created the Italian national health service (NHS) and was designed to rectify the existing fragmentation of health care has never been completely translated into practice. This is partly a result of the resistance and vested interests of different agencies and groups. In 1993, a law which introduced "managed competition" was passed, but its ability to solve some of the service's major problems is still uncertain. There are concerns about the efforts that managed competition or a market economy might have on the profound inequalities that already exist between different geographical areas, and especially between the north and the south of Italy. This concern is particularly valid if the financial changes are not accompanied by a sound epidemiological perspective - a perspective which has traditionally been neglected in Italy.

The crisis in the Italian NHS is not very different from that being experienced by other industrialized countries, but there are some aspects peculiar to Italy. In the past, the policy making process has been based on more political aspects than technical studies - there is no culture that places high value on research in policy decisions. Establishing a link between science and policy making started to be seen as a necessary step only recently, when the various parts of the health care system became involved in dialogue.

This paper aims to describe some key issues concerning the health status of the Italian population and the structure of health care in Italy in order to pinpoint the most critical aspects that could be seriously threatened by future policies. The ultimate aim is to highlight the potential contribution of epidemiology - neglected until now - in the approach to the NHS crisis. Special emphasis will be placed on the north/south differences. In fact, both the epidemiology of common diseases and the functioning of health services differ in the different parts of Italy, and they require separate comments. For a long time southern Italy has had a poorer economy than the northern regions. In addition, state intervention has been devoted more to avoiding massive unemployment than to assuring an effective and efficient use of public resources.

Living conditions and public health: some examples

According to the European Atlas of Avoidable Deaths, ${ }^{12}$ while Italian mortality rates for most avoidable causes of death are close to the mean European values, this is not the case for other causes. In particular, southern regions show mortality rates for rheumatic cardiopathy (age 5-44) which are two to four times higher than the European mean. In addition, deaths from cervical cancer are considerably higher in southern regions (Sicily, Puglia, Calabria).

While excesses for both rheumatic cardiopathy and cervical cancer indicate the existence of chronic hygiene problems, the organisation of health care may also be relevant. There is evidence that the uneven distribution of deaths from rheumatic cardiopathy is at least partly a consequence of poor management of patients with rheumatic fever, rather than of a different incidence of rheumatic fever itself. In the case of cervical cancer, the availability of 
the Pap smear is not the same across Italian regions. According to a survey, the number of Pap tests per 100 woman-years in 1986 was 22.5 in northern regions but only 10.8 in the south. ${ }^{3}$

\section{Uneven distribution of high technology} Advanced diagnostic equipment is clearly unevenly distributed throughout Italy. The availability of computed tomography is approximately 1 scanner per 110000 inhabitants in northern and central regions, and 1 per 172000 inhabitants in the south. Magnetic resonance equipment has a density of 1 per 500000 inhbitants in the north versus 1 per 900000 population in the south. Density values reach 1 per 500000 (north) and 1 per 1200000 inhabitants (south) in the case of linear accelerators (data refer to 1992). (Italian Department of Health, unpublished data, 1993.)

This uneven distribution does not necessarily mean that there is a shortage of this technology. The case of lithotripters is paradigmatic. They are about 1 per 500000 inhabitants in each of the large Italian areas, a density which is much more favourable than in most European countries. In the UK, for example, there was 1 lithotripter per 3770000 inhabitants in 1989 , in Denmark 1 per 1700 000, and in France 1 per 1500000 - in a period in which the rate was already 1 per 800000 in Italy. ${ }^{4}$ Most lithotripters $(58 \%)$ in Italy are, however, in the private sector. ${ }^{4}$ Paradoxically, in parallel with this ready availability of heavy technology there is large underuse of it. In most public hospitals expensive machinery is used for only a few hours in the morning.

\section{Distribution of private and public hospitals}

The private sector is more developed in southern Italy than in the north. For example, 26\% of the hospital beds in Campania and $34 \%$ in Lazio are in private hospitals compared with $13 \%$ in Piedmont and $16 \%$ in Lombardy. ${ }^{5}$ The private sector has a very special status in Italy, however, since it has been strongly linked with the public sector until now. According to agreements between the NHS and private hospitals or laboratories, patients who cannot be accepted within the public sector - for example, because of long waiting lists - are treated by private institutions, which are then refunded by the NHS. This type of relationship between public and private structures has obviously nothing to do with "managed competition". Rather, it results in a reduction in productivity of the public sector for at least two reasons. Firstly, the public sector is not motivated to solve its problems, for example waiting lists. In some instances, there is evidence that public sector waiting lists become longer and longer just to allow private clinics to increase their activities. Secondly, many physicians and nurses (particularly in southern regions) work in both a public and in a private hospital. This phenomenon leads to a poor use of resources, since public equipment and structures (in par- ticular surgical theatres) are used for only a limited time. Both the distribution of private hospitals in the south and their very special financial relationships with the public sector are the consequence of a strong political rather than scientific influence on decision making. Southern regions have more private hospitals for reasons that include: the lack of a tradition of efficient public services; the speedy and unplanned development of medicine in the postwar period; and the intertwining of national politics and local groups of medical management. This picture is not peculiar to the health sector, however, it is widespread in all public services in southern Italy.

\section{Clinical variability: the example of breast cancer}

There is evidence that Italy is affected by considerable inter-regional variation in clinical practice. Clinical management of breast cancer has been studied extensively and shown to be subject to large variation. One of the most striking results of a recent survey on 1724 breast cancer patients was the finding that in two southern regions the proportions of women with breast cancer who had undergone termography (a completely obsolete examination) were $26 \%$ in Puglia and $14 \%$ in Sicily, while in northern regions proportions ranged between 1 and $8 \% .^{6}$ An index of completeness and appropriateness of the diagnostic procedures, ranging from 0 to 100 , ranged from a minimum of 40 in Calabria to a maximum of 89 in the Aosta Valley. In addition, the therapeutic management of patients is often questionable: the proportion of patients undergoing appropriate surgical intervention ranged between $52 \%$ in Calabria and $88 \%$ in Umbria. ${ }^{6}$

As far as mammographic screening is concerned, in 1991 there were 226 mammography units in public hospitals and 50 in private hospitals in the northern regions, 108 and 23 respectively in regions of central Italy, and 66 and 79 in southern regions. ${ }^{7}$ It has been estimated, according to international protocols for mammographic screening, that the number of units is generally sufficient in the northern and central regions but that southern regions need 167 instead of the existing 145 units (1991 estimates). Also, the compliance of women in screening programmes is far from complete and evenly distributed. According to surveys, in northern regions less than 1 in 10 women (age 18-69 years) had undergone mammography at least once. This estimate is certainly much lower in the south. However, the situation in this particular area is changing rapidly.

The disturbing implication of practice variation is that differences have been proved to represent suboptimal or harmful care for some patients and, at the same time, an inappropriate use of resources for many others. Research in Italy has shown that: (1) overuse occurs in small areas or patient groups, or both; (2) underuse of appropriate care is as much a problem as overuse and has important implications in terms of avoidable morbidity, mortality, and cost. ${ }^{8}$ 


\section{Quality assurance}

Quality assurance is one of the key issues in the reform law passed in 1993. For a long time, Italy has had only the kind of quality control that was implied by the licensing and registration procedures. Currently, assessment of efficacy and safety of health technologies is pursued before their approval for use, while monitoring of efficiency in their use is rarely done. In 1992, a national programme encompassing a system of quality assurance committees was launched by the Department of Health. Regional committees have been appointed in only 13 of the 21 Italian regions, however, and specific programmes of quality assurance have been reported from fewer than $14 \%$ of the 700 local health units. One key issue is that quality assurance should be aimed at improving health care effectiveness, and not just focussing on managerial efficiency. Efficiency pertains mostly to the optimal combination of resources and implies that we already know what good medical practice is. Very limited experience has been achieved in Italy with the organisation of consensus conferences and the development of guidelines. This is the necessary path, however, if effective quality assurance is to be pursued. ${ }^{8}$ Apart from the emphasis on efficiency rather than effectiveness, quality assurance in Italy has been mainly perceived as a routine activity with no real impact on the organisation and functioning of health services.

\section{Discussion}

Recent legislative changes are intended to introduce "managed competition" into the Italian national health service and to promote quality assessment within its structures. Until now there has been a wide gap between the clinical world, epidemiological research, the development and distribution of new medical technologies, and health administration. The policy making process has largely been based on a "muddling through" mentality; the process of decision making has not been easily traceable and, therefore, it has been difficult to criticise. Confusion is made traditionally between technical choices, social goals, and politics. Politics, in itself, has been largely dominated by vested interests and corruption until very recently.

In the past three years in Italy a few important meetings have been devoted to the theme of technology assessment, including the 1993 meeting of the International Society for Technology Assessment in Health Care. These meetings involved epidemiologists, clinicians, and health administrators. The meetings of the Italian Society for Quality Assessment in Health Care (October 1992) and the Italian Association of Epidemiology (AIE) (May 1993) concerned specifically the quality and effectiveness of health care in Italy. At the AIE meeting the main presentations were about effectiveness of care in neurology, cardiology, oncology, neonatology, obstetrics, drug addiction, psychiatry, and occupational health. The speakers were invited to summarise the evidence on both clearly effective and clearly ineffective treatments in each discipline, to suggest areas which require further research, and to identify discrepancies between scientific evidence and diffusion in medical practice. There is, in fact, considerable evidence that clinical practice in Italy is very variable - that ineffective care is still practised and that effective care has not been completely adopted. ${ }^{9}$

The involvement of epidemiologists in issues such as evaluation of effectiveness of care, consensus conferences, and guidelines for clinical practice would greatly contribute to bridging the gap between science, clinical practice, and health administration in Italy. To reach such a goal, however, the government and the Ministry of Health must understand the potential of epidemiology and related disciplines and set up the necessary structures. One example of the latter is the Office for Technology Assessment in Health Care which has been organised in the Veneto region, the first in Italy of this type. A regional structure of such offices would be highly desirable, both because the new law increases considerably the power of the regions in planning health care and allocating resources and because there are strong regional differences and specificities in health care problems. Obvious problems that should be addressed by such offices, together with the Ministry of Health, are the following:

- The uneven distribution of medical technologies in Italy;

- The persistence of large inequalities, documented by outcomes such as rheumatic fever, between northern and southern regions;

- The large variability in clinical practices;

- The lack of an explicit policy as far as the distribution of high technologies is concerned;

- The ensuing "hidden" rationing, since official criteria do not seem to be used for the recruitment of eligible patients; and

- The peculiar relationship between public and private health structures, which does not contribute to an increase of productivity through competition.

This is only a short list of problems which clearly require an epidemiological assessment.

Unfortunately, while the development of epidemiology has been speedy in the past two decades in Italy, it has been very disorganised. Epidemiological research has reached international standards, but there is a serious lack of sound epidemiological teaching in medical schools. The epidemiology taught at the universities is often old fashioned (infectious disease epidemiology), largely disconnected from both public health needs and the clinical world, and (with few exceptions) teachers have little research experience.

From a positive perspective, the health care reform creates special opportunities to experiment in order to solve some of the major problems of the Italian NHS. Positive features of the Italian system include: a widespread network of public services with full time personnel, allowing an ongoing quality assurance; the regionalised administration, which should 
avoid some of the drawbacks associated with a centralised system; the emphasis in the recent law on quality assurance; and the official involvement of patients and their organisations in the identification of problems and the proposal of solutions. We hope that these opportunities will not be missed and that the positive features of the new law will not be totally overwhelmed by emphasis on privatisation and "the market".

We thank Renaldo Battista, Carlo Faveretti, Richard Hayes, and Francesco Taroni for fruitful comments.

1 Holland WW ed. European Community atlas of avoidable death. Oxford, Oxford University Press/Council of Economic Communities, 1988

2 Morosini PL, Lauriola P, Magliola E, Feola G, Paul EM. Le morti evitabili nella valutazione dell'attività del servizio sanitario. Epidemiologia e Prevenzione 1990;45:48-58.

3 Ferraroni M, La Vecchia C, Pagano R, Negri E, Parazzini F, Decarli A. Patterns of cervical screening utilization in Italy. Tumori 1989;75:521-6.

4 Dirindin N, Vanara F. The diffusion of expensive medical technologies in Italy. Proceedings, International Society of Technology Assessment in Health Care. Sorrento May 23-26, 1993.

5 Consiglio Sanitario Nazionale. La salute degli italiani - 1988. Roma: Consiglio Sanitario Nazionale, 1990.

6 Grilli R, Apolone A, Confalonieri C, Nicolucci A, Scorpiglione N, Zola P, Liberati A. La qualità dell'assistenza nelle pazienti con carcinoma della mammella negli anni 1979-1987. Federazione Medica 1991;XLIV(3):161-7.

7 Franceschi S, Geddes M. La prevenzione dei tumori femminili. In: Geddes $M$ ed. La salute degli italiani, rapporto 1991. Roma: La Nuova Italia Scientifica, 1991.

8 Taroni F. NHS and quality assurance in Italy. Proceedings: Issues in public health medicine. Rome June 30, 1993.

9 Taroni F, Tognoni G, Di Lallo D, Davoli M, Ricci S, Ruggeri M, Baldasseroni A. Effectiveness of medical care. Proceedings: 17th Meeting of the Italian Association of Epidemiology. Epidemiologia e Prevenzione 1994;18:69-118. 